\title{
MEDICINAL HERBS OF PASIR MAYANG, JAMBI: ETHNOPHARMACY AND TOXICITY SCREENING
}

\author{
HILMAN AFFANDI*, ARIF NURYADIN* and SUSILO B. PRAYOGO* \\ *SEAMEO BIOTROP, Jl. Raya Tajur Km. 6 \\ Bogor, Indonesia
}

\begin{abstract}
This article presents the results of an investigation concerning the use of herbal medicinal plants by the people of Pasir Mayang, sub-District (Municipality) of VII Koto, District of Tebo, Jambi Province, Sumatera. The data collection was based on interviews with the healers and other villagers of Pasir Mayang who possess knowledge of the different plants and their medicinal uses. The study recorded 57 species of medicinal plants used in Pasir Mayang. The detailed uses of the 57 medicinal plants are given. All plant species were subjected to phytochemical analysis and toxicity tests, and the outcome of the analysis on the presence of alkaloids, saponins, steroids/terpenoids, and the level of toxicity against brine shrimp (Anemia salina) are presented. A comparison with other studies reported in the literature seems to indicate that a high frequency of the use of leaves in therapy may be a part of a larger cultural phenomenon among the tropical forest tribes of Southeast Asia.
\end{abstract}

Keywords : Indonesia/Jarabi/Pasir Mayang/medicinal plants/ethuophannacy/toxicity screening

\section{INTRODUCTION}

Pasir Mayang is the name of an area in the District of Tebo in the province of Jambi. In the beginning, the name of Pasir Mayang was Dusun Tuo. However, since the establishment of PT Industries et Forest Asiatiques (IFA), it has used the name Pasir Mayang. In line with the increasing population and then later, with the influx of settlers from neighboring communities, Pasir Mayang developed to become several villages which were administratively included in the sub-district of VII Koto.

Pasir Mayang can be reached by road from the District of Muara Bungo, traversing about 20 $\mathrm{km}$, then turning right at the Simpang Sawmill for about another $20 \mathrm{~km}$ on the way to the Transmigration Area of Rimbo Bujang to the village of Dusun Tuo. From there, the sub-district VII Koto can be reached by continuing $8 \mathrm{~km}$ more. The access to the village of Sungai Karang can be reached from Dusun Tuo through unpaved roads traversing $3 \mathrm{~km}$ to Batanghari River, then crossing the Batanghari River by boat and taking another $15 \mathrm{~km}$ more of unpaved roads to the village of Sungai Karang.

The sub-district VII Koto covers an area of 112,700 ha bordering the province of West Sumatera in the northern side. The southern part of the area is bounded by the sub-district of Tebo Ulu, and the western side by the District of Bungo. Meanwhile, the eastern side is bounded by the Province of Riau. 
The population of the sub-district VII Koto, based on a census conducted by the office of subdistrict VII Koto on April 2001, is composed of 19,249 individuals which consisted of 9,595 men, and 9,654 women, comprising over 11 villages.

Forest is no longer a source of income for the people of Pasir Mayang. The forest in the area has been used for collecting a wide range of forest fruits and medicinal plants, not to mention building materials and firewood. Occasionally, people collect rattan from the forest, but even this is hard to find, because lots of rattan had been collected for a long time for commercial purposes. While the people of Pasii Mayang are not "primitive" forest-dwellers, they have retained a relatively simple agricultural way of life. Therefore, agriculture is the main occupation of the people of Pasir Mayang. Traditional farming (ladang) usually takes place far away from the villages and this usually involves one-quarter to one-half hectare of forest which are cleared and burned and planted with a wide variety of crops. Most of their harvest, however, are adequate only to supply their own needs.

The cultivation of "pohon karet" or rubber tree (Hevea brasiliensis) is important in Pasir Mayang as one of the main sources of income in this otherwise moneyless society. Rubber is planted in the forest close to the villages. At certain ages of the rubber trees, farmers tap and collect the latex, and sell them to the collectors in the district of VII Koto or Tebo. They also plant coffee in the forest for their own use or sell to the market.

The health center, more widely known as Puskesmas, is located in the sub-district of VII Koto, headed by a medical doctor, assisted by another medical doctor, one dentist and eight paramedical personnel. Health services are provided free of charge for those who could provide proof that they are not able to pay. A Bidan (nurse) is available in each village. Nurses are trained to diagnose basic illnesses and diseases and can provide a number of common medicines. These common generic medicines which are subsidized by the government with generic names, are supplied every three months from the Health Center in the sub-district of VII Koto and are given free of charge. Pembantu Puskesmas (health sub-centers) exist in each village in the sub-District of VII Koto, headed by a nurse and some paramedical personnel.

The most prevalent illnesses are diarrhea, cholera and malaria. Meanwhile, other communicable diseases include leprosy, framboesia, tetanus neonatorum, diphtheria, whooping cough, and filiariasis. No smallpox cases have been registered. Due to the proximity of the area, and also on account of scarcity of medicine, doctors could not conduct regular visits to villages, which are far from the sub-District of VII Koto. Only when an epidemic occurs or the situation becomes really threatening medical assistance will be sent. The distance between the district and the health centers, as well as the high expenses which travel incurs are the main obstacles preventing the population from making use of the health facilities provided by the government for them.

People generally accept the modern medicines that provide wide therapeutic measures for illnesses and diseases that people suffer from, except for mental illness. 
For this group of diseases, people will then ask for help directly from the traditional healers. In much the same way, if health problems persist and cannot be solved by the health center, they will then turn to traditional healers who combine herbal remedies with mystical ceremonies which, according to those interviewed, counter the influence of what was generally believed to be "evil spirits" which are responsible for the illnesses, diseases, or even accidents the citizens experience.

Almost all cultures have a long history of folk or traditional medicine, which includes the use of medicinal plants or herbs prescribed by local folk or traditional doctors in the neighborhood. Even in ancient cultures, people methodically and systematically collected information on herbs and developed well-defined herbal pharmacopoeias. This must have also occurred to the ancient people of Pasir Mayang, for there are still quite a number of traditional healers who do their ritual practices in the communities.

This study provides an outline of the contemporary knowledge on the use of herbal medicinal plants by the community around Pasir Mayang with the purpose of preserving this aspect of traditional knowledge for the generations to come.

Specifically, this research aims to make an inventory of herbal medicinal plants available in Pasir Mayang and how these are used by the community residents. It further aims to describe the herbal plants used for medicinal treatment by the indigenous people paying particular attention to the methods of preparation prior to its use, identification of the specific illness or disease that can be treated by each plant, and the effect of the treatment. Phytochemical analysis and toxicity tests had also been conducted on the medicinal plants collected in the area, to determine the toxic level of the methanolic plant extracts against brine shrimp (Artemia salina) and to trace the presence of specific chemical substances such as alkaloids, steroids, saponins, and flavonoids.

\section{METHODOLOGY}

\section{Data collection of Pasir Mayang medicinal plants}

Information on the traditional medicinal plants and herbs in Pasir Mayang was collected by a team of four workers in a duration of two months in the year 2003, in April and October. Information was obtained for the series of one-hectare plots. In each case, one-strip transects $(25 \mathrm{x}$ $100 \mathrm{~m}$ ) were made within each plot marked out by coloured plastic ropes at both ends and in the centre. Inputs were collected in the presence of local healers or other villagers by walking along the centre line. The villagers were asked to identify the local names and uses of each plant occurring within the series of transects. For every plant with indication of traditional use(s), every information was recorded and herbarium specimens of each species were collected by pressing between newsprints, stacked and placed in heavy plastic bags, treated with denatured alcohol and packed, opened only upon arrival at the SEAMEO BIOTROP laboratory in Bogor. Small amounts of plant materials from 
each plant were taken to the SEAMEO BIOTROP natural products laboratory for phytochemical analysis carried out at a later date.

\section{Toxicity test (brine shrimp lethality bioassay)}

Twenty grams of air-dried plant materials from each species were extracted with methanol at room temperature. The crude extracts were evaporated under reduced pressure until dried. Sea water was poured into a small tank and shrimp eggs were added to the tank provided with aerator. After two days the shrimp eggs hatched and mature as naupili. Each crude extract was weighed (20 mg) and dissolved with dichloromethane (2 ml). From this solution, 500, 50, 5ul were transferred to small petri dish corresponding to 1000, 100, 10 (J.g/ml (ppm), respectively (Meyer et al. 1982). The solvent was evaporated by standing overnight at ambient temperature. After two days, when the shrimp larvae were ready, sea water was added to each petri dish containing 10 shrimps per dish (30 shrimps per dilution), and adjusted the volume with sea water to $5 \mathrm{ml} / \mathrm{dish}$. The number of shrimps that survived were counted and recorded after 24 hours. The data were analyzed with Finney computer program to determine $\mathrm{LC}_{0}$ values at $95 \%$ confidence intervals (Hostettmann 1991). Each crude extract of the sample was tested in three replicates with one control each. The controls were treated in a similar manner without the presence of the crude extracts.

\section{Phytochemical analysis}

Alkaloid testing was conducted according to procedures described by Culvenor and Fitzgerald (1963) : two to four leaves, fruits and/or bark of each plant of the collected species were preserved in ethanol, after being dried in the laboratory, grounded with clean sands and added with $10 \mathrm{ml}$ chloroform. Ammoniacal chloroform (10 ml, 0.05 M) was added, stirred and filtered, then shaken with aqueous sulphuric acid (10 drops, $2 \mathrm{M})$. Then the aqueous layer was tested with Mayer's reagent. A dense, heavy precipitate was designated as $3+$, strong precipate as $2+$ and weak precipate as $1+$.

Terpenoids/steroids and saponins were screened on the basis of Liebermann-Burchard and froth test, respectively, as generally described by Simes et al. (1959). An amount of 2-4 g dry leaves were cut into small pieces and boiled in ethanol (25 ml, $15 \mathrm{~min}$.), filtered while still hot and evaporated to dryness. The extract was triturated with ether and the insoluble ether shaken with water (ca. $5 \mathrm{ml}$ ) in a $\mathrm{Vi}$ x 5-inch test tube. A foam more than $3 \mathrm{~cm}$ and lasting for more than 15 minutes was designated as $3+$, between $2-3 \mathrm{~cm}$ was designated as $2+$ and foam of $1-2 \mathrm{~cm}$ was designated as $1+$. The ether soluble fraction was subjected to the Liebermann-Burchard test using acetic anhydride and sulphuric acid. The formation of a bright purple, red or pink coloration was considered 3+ while moderate and weak colora- 
tion were designated $2+$ and $1+$, respectively. In the case of doubtful coloration produced, the ethereal fraction was passed through a Pasteur pipette filled with active charcoal just enough to absorb chlorophyll and treated as above.

\section{RESULTS AND DISCUSSION}

A total of 57 herbs were collected and noted according to their uses for various purposes by the healers and villagers of Pasir Mayang. Of these, the medicinal uses which were presumed magical or superstitious in nature were considered of no interest for the present study and have been omitted for further discussions. Many of these comprise common weedy species and a few cultivated ones, all of which were easily available, in those areas where this study was conducted, as well as in most other villages in Pasir Mayang (Affandi et al. 1996). Many of the plants, however, were of forest origin, growing in the secondary and little-disturbed forests in the surrounding areas.

In the list, species were arranged alphabetically by family/followed by common name / plant part(s) used / and a description of their uses. These were summarized in Appendix 1.

\section{Medicinal Uses}

The process to cure common maladies and illnesses among the people of Pasir Mayang involves accessing both traditional and modern methods. Generally, the people of Pasir Mayang rely mostly on traditional medicines to treat minor ailments or are used as first aid towards a cure. They seek " modern " medicine only when the traditional methods have been exhausted.

The most frequently utilized plant part is the leaf (Table 1). This preference for leaves is apparently derived from the fact that therein, many species store high concentrations of bioactive compounds (Moore 1994). In addition to efficacy, other factors that may contribute to the preference for leaves as medicine include the ease with which they may be collected, stored, and transported and the ease with which bioactive compounds may be extracted.

The most frequently cited modes of preparation (Table 2) were: poultice (mashed, crushed, or chopped plant part), decoctions (boiling of plant parts), and used directly from the plants (none).

The most frequently encountered modes of administration are oral and topical (Table 3) which may be preferred because they are believed to be the most effective means for delivering bioactive compounds into the body. 
BIOTROPIA No. 22, 2004

Table 1. Parts of medicinal plants used by Pasir Mayang people

\begin{tabular}{lc}
\hline \hline Part used & Total \\
\hline Leaf & 44 \\
Fruit & 3 \\
Root & 6 \\
Flower & 2 \\
Bulb & 1 \\
Stem & 4 \\
\hline
\end{tabular}

Table 2. Mode of preparation frequently used for Pasir Mayang medicinal plants

\begin{tabular}{lc}
\hline \hline Mode & Total \\
\hline Poultice & 34 \\
Decoction & 19 \\
None & 8 \\
\hline
\end{tabular}

Table 3. Mode of administration of medicinal preparations by Pasir Mayang people

\begin{tabular}{lc}
\hline \hline Mode & Total \\
\hline Oral & 34 \\
Topical & 23 \\
Bath & 4 \\
Inhalation & - \\
\hline
\end{tabular}

\section{Phytochemical Analysis and Toxicity Tests}

The phytochemical analysis is a preliminary test to trace the presence of specific chemical substances such as alkaloids, steroids/triterpenes, and saponins without resulting to a biological screening. A total of 57 species from 30 families were phytochemically tested in the Laboratory of Natural Products at SEAMEO BIOTROP. The results of the tests are summarized in Table 4. 
Table 4. Phytochemical Analysis and Toxicity Tests of Plant Extracts of the Medicinal Plants Collected from Pasir Mayang

\begin{tabular}{|c|c|c|c|c|c|c|c|}
\hline No & Species & Local Name & $\begin{array}{l}\text { Part } \\
\text { used }\end{array}$ & Alkaloid & Saponin & $\begin{array}{l}\text { Steroid/ } \\
\text { Tri } \\
\text { terpenoid }\end{array}$ & $\begin{array}{c}\text { Toxicity } \\
\text { LC }_{50} \\
\text { (i.c. } 95 \%)\end{array}$ \\
\hline 1 & $\begin{array}{l}\text { Acanthaceae } \\
\text { Justicia gendarusa } \\
\text { Linn. }\end{array}$ & Setajam & $\mathbf{L}$ & - & $1+$ & $2+$ & $>1000$ \\
\hline 2 & $\begin{array}{l}\text { Amaranthaceae } \\
\text { Calosia argentea } \\
\text { Linn. }\end{array}$ & Bunga merah & L & - & $2+$ & $2+$ & 645 \\
\hline 3 & $\begin{array}{l}\text { Amaryllidaceae } \\
\text { Curculigo capitulata } \\
\text { O.K. }\end{array}$ & $\begin{array}{l}\text { Ketari/Katorih } \\
\text { bareh }\end{array}$ & L & - & - & $1+$ & $>1000$ \\
\hline 4 & $\begin{array}{l}\text { Curculigo latifolia } \\
\text { Dryand }\end{array}$ & Benit & L & - & $1+$ & $2+$ & $>1000$ \\
\hline 5 & $\begin{array}{l}\text { Asclepidirceae } \\
\text { Cryptolepis javanica } \\
\text { BI. }\end{array}$ & Akar daun taun & L & - & $1+$ & $3+$ & $>1000$ \\
\hline 6 & Hoya sp. & Daun setebal & L & - & $1+$ & $3+$ & 875 \\
\hline 7 & $\begin{array}{l}\text { Asteraceae } \\
\text { Blumea balsamifera } \\
\text { DC. }\end{array}$ & Celapo tai & L & - & $2+$ & $3+$ & $>1000$ \\
\hline 8 & $\begin{array}{l}\text { Burseraceae } \\
\text { Canarium littorale } \\
\text { B1. }\end{array}$ & Ampelas licin & L & - & $1+$ & $2+$ & $>1000$ \\
\hline 9 & $\begin{array}{l}\text { Connaraceae } \\
\text { Roureopsis javanica } \\
\text { Planch. }\end{array}$ & Akar jaring tupai & L & - & - & $2+$ & $>1000$ \\
\hline 10 & $\begin{array}{l}\text { Cyatheaceae } \\
\text { Hemitelia latebrosa } \\
\text { Mett. }\end{array}$ & Pakis longgok & L & - & $2+$ & $1+$ & $>1000$ \\
\hline 11 & $\begin{array}{l}\text { Dioscoreaceae } \\
\text { Dioscorea aculeata } \\
\text { Linn. }\end{array}$ & Kabenar & L & - & $2+$ & $1+$ & $>1000$ \\
\hline 12 & $\begin{array}{l}\text { Euphorbiaceae } \\
\text { Melanolepis } \\
\text { multiglandulosa } \\
\text { Reich.ex Zoll. }\end{array}$ & Bunga sepanggil & $\mathbf{L}$ & - & $1+$ & $1+$ & $>1000$ \\
\hline 13 & $\begin{array}{l}\text { Gleicheniaceae } \\
\text { Gleichenia linearis } \\
\text { Clarke }\end{array}$ & Resam & $\mathbf{L}$ & - & $1+$ & $1+$ & 143 \\
\hline 14 & $\begin{array}{l}\text { Graminaceae } \\
\text { Centotheca latifolia } \\
\text { Trin. }\end{array}$ & $\begin{array}{l}\text { Rambut pulut- } \\
\text { pulut }\end{array}$ & L & - & $1+$ & $2+$ & $>1000$ \\
\hline
\end{tabular}


Table 4. Continued

\begin{tabular}{|c|c|c|c|c|c|c|c|}
\hline No & Species & Local Name & $\begin{array}{l}\text { Part } \\
\text { used }\end{array}$ & Alkaloid & Saponin & $\begin{array}{l}\text { Steroid/ } \\
\text { Tri } \\
\text { terpenoid }\end{array}$ & $\begin{array}{l}\text { Toxicity } \\
\mathrm{LC}_{50} \\
\text { (i.c. } 95 \% \text { ) }\end{array}$ \\
\hline 15 & $\begin{array}{l}\text { Driockloa djainkareh } \\
\text { Bush. }\end{array}$ & Buluh hitam & L & & $1+$ & $1+$ & $>1000$ \\
\hline 16 & $\begin{array}{l}\text { Gigantochloa } \\
\text { hasskarliana Becc. }\end{array}$ & Bambu nipis & L & - & $1+$ & $1+$ & $>1000$ \\
\hline 17 & $\begin{array}{l}\text { Schizostachyum } \\
\text { zollingeri Kurz. }\end{array}$ & Buluh nipis & $\mathbf{L}$ & - & $1+$ & $2+$ & $>1000$ \\
\hline 18 & $\begin{array}{l}\text { Labiatae } \\
\text { Ocimum basilicum } \mathrm{L} \text {. } \\
\text { Leguminosae }\end{array}$ & Selasih A & $\mathrm{L}$ & - & $1+$ & $2+$ & $>1000$ \\
\hline 19 & Cassia alata & ketepeng & $\mathrm{L}$ & - & $1+$ & $2+$ & $>1000$ \\
\hline 20 & $\begin{array}{l}\text { Milletia scicea W.et } \\
\text { Liliaceae }\end{array}$ & Akar semambu & $\mathrm{L}$ & - & $1+$ & $2+$ & $>1000$ \\
\hline 21 & Dianella montana B1. & $\begin{array}{l}\text { Batang sia/ } \\
\text { Akar sayak }\end{array}$ & $\mathbf{L}$ & - & $1+$ & $1+$ & $>1000$ \\
\hline 22 & $\begin{array}{l}\text { Smilax leucophylla } \\
\text { Bl. }\end{array}$ & Akar gunung & L & - & $1+$ & $1+$ & $>1000$ \\
\hline 23 & $\begin{array}{l}\text { Mucuna gigantean } \\
\text { DC }\end{array}$ & Akar katai & $\mathrm{R}$ & - & $1+$ & $2+$ & $>1000$ \\
\hline & Menispermaceae & & & & & & \\
\hline 24 & Fibraurea sp. & Akar kunyit & $\mathrm{R}$ & $4+$ & $1+$ & $2+$ & 608 \\
\hline 25 & $\begin{array}{l}\text { Percamphylus } \\
\text { glaucus Merr. }\end{array}$ & $\begin{array}{l}\text { Akar limpanang/ } \\
\text { Akar manang/ } \\
\text { Akar benang }\end{array}$ & $\mathrm{L}$ & - & $1+$ & $2+$ & $>1000$ \\
\hline 26 & $\begin{array}{l}\text { Monimiaceae } \\
\text { Kibarasp. } \\
\text { Piperaceae }\end{array}$ & Udu karo & $\mathrm{L}$ & - & $1+$ & $1+$ & $>1000$ \\
\hline 27 & Piper aduncum Lind. & Kayu sirih & $\mathrm{L}$ & - & $1+$ & $3+$ & $>1000$ \\
\hline 28 & Piper betle Linn. & Sirih & $\mathrm{L}$ & - & $2+$ & $2+$ & $>1000$ \\
\hline 29 & Piper sp. & Akar sirih duduk & $\mathrm{L}$ & - & $1+$ & $2+$ & $>1000$ \\
\hline 30 & $\begin{array}{l}\text { Piper sp. } \\
\text { Polypodiaceae }\end{array}$ & Sirih antu & L & - & $1+$ & $2+$ & 295 \\
\hline 31 & $\begin{array}{l}\text { Aspodium ternatense } \\
\text { A.v.R. }\end{array}$ & Kelakap & L & - & $1+$ & $3+$ & $>1000$ \\
\hline
\end{tabular}


Table 4. Continued

\begin{tabular}{|c|c|c|c|c|c|c|c|}
\hline No & Species & Local Name & $\begin{array}{l}\text { Part } \\
\text { used }\end{array}$ & Alkaloid & Saponin & $\begin{array}{l}\text { Steroid/ } \\
\text { Tri } \\
\text { terpenoid }\end{array}$ & $\begin{array}{c}\text { Toxicity } \\
\text { LC }_{50} \\
\text { (i.c. } 95 \%)\end{array}$ \\
\hline 32 & $\begin{array}{l}\text { Dictyopteris } \\
\text { irregularis } \operatorname{Pr} .\end{array}$ & Paku limat & $\mathbf{L}$ & $1+$ & $1+$ & - & 2 \\
\hline 33 & $\begin{array}{l}\text { Dryopteris boryana } \\
\text { O.Chr. }\end{array}$ & Pakis longgoh & $\mathbf{L}$ & - & $2+$ & $1+$ & $>1000$ \\
\hline 34 & $\begin{array}{l}\text { Lomariopsis } \\
\text { speciabilis }\end{array}$ & Pakis gajah & $\mathrm{R}$ & - & $1+$ & $1+$ & $>1000$ \\
\hline \multirow[t]{2}{*}{35} & $\begin{array}{l}\text { Nephrolepis } \\
\text { hirsutula Presl. }\end{array}$ & Pakis & L & - & $1+$ & $1+$ & $>1000$ \\
\hline & Rhamnaceae & & & & & & \\
\hline \multirow[t]{2}{*}{36} & Zizyphus sp. & Akar kait & $\mathrm{L}$ & $3+$ & $1+$ & $2+$ & $>1000$ \\
\hline & Rhyzoporaceae & & & & & & \\
\hline \multirow[t]{2}{*}{37} & $\begin{array}{l}\text { Anisophyllea disticha } \\
\text { Baill. }\end{array}$ & $\begin{array}{l}\text { Batang ibu/ } \\
\text { Batang beribu }\end{array}$ & $\mathbf{L}$ & - & $2+$ & $2+$ & $>1000$ \\
\hline & Rubiaceae & & & & & & \\
\hline 38 & Lxora javanica DC. & Kayu telor & L & - & $2+$ & $1+$ & $>1000$ \\
\hline 39 & Uncaria sp. & Akar kait & $\mathbf{L}$ & $3+$ & $1+$ & $2+$ & 654 \\
\hline \multirow[t]{2}{*}{40} & Uncaria sp. & Akar kukualang & $\mathrm{L}$ & - & $1+$ & $2+$ & $>1000$ \\
\hline & Rutaceae & & & & & & \\
\hline \multirow[t]{2}{*}{41} & $\begin{array}{l}\text { Clausena excavata } \\
\text { Burm. }\end{array}$ & $\begin{array}{l}\text { Daun secere/ } \\
\text { Cere-cere/ Kayu } \\
\text { angit }\end{array}$ & $\mathbf{L}$ & $1+$ & $1+$ & $1+$ & $>1000$ \\
\hline & Schizophyceaceae & & & & & & \\
\hline \multirow[t]{2}{*}{42} & $\begin{array}{l}\text { Lygodium circinatum } \\
\text { Sw. }\end{array}$ & $\begin{array}{l}\text { Akar kawe/ } \\
\text { Akar sapoi/ } \\
\text { Akar menang }\end{array}$ & $\mathrm{L}$ & - & - & $2+$ & 999 \\
\hline & Selaginellaceae & & & & & & \\
\hline \multirow[t]{2}{*}{43} & $\begin{array}{l}\text { Selaginella plana } \\
\text { Hieron }\end{array}$ & $\begin{array}{l}\text { Sigagar } \\
\text { tegak/Paku } \\
\text { anai-anai/ Kuku } \\
\text { bener/ Nganai } \\
\text { duduk }\end{array}$ & $\mathrm{L}$ & - & $3+$ & - & $>1000$ \\
\hline & Solanaceae & & & & & & \\
\hline 44 & Solanum torvum Sw. & Dubang & L & - & $2+$ & $3+$ & $>1000$ \\
\hline
\end{tabular}


BIOTROPIA No. 22, 2002

Table 4. Continued

\begin{tabular}{|c|c|c|c|c|c|c|c|}
\hline No & Species & Local Name & $\begin{array}{l}\text { Part } \\
\text { used }\end{array}$ & Alkaloid & Saponin & $\begin{array}{c}\text { Steroid/ } \\
\text { Tri } \\
\text { terpenoid }\end{array}$ & $\begin{array}{l}\text { Toxicity } \\
\text { LC }_{50} \\
\text { (i.c. } 95 \%)\end{array}$ \\
\hline & Verbenaceae & & & & & & \\
\hline 45 & $\begin{array}{l}\text { Clerodendron inerma } \\
\text { Gaertn. }\end{array}$ & Bunga pekan & $\mathrm{L}$ & - & $2+$ & $2+$ & 282 \\
\hline 46 & $\begin{array}{l}\text { Clerodendron } \\
\text { squamatum Vahl. }\end{array}$ & Bunga panggil & $\mathrm{L}$ & - & $1+$ & $1+$ & $>1000$ \\
\hline 47 & $\begin{array}{l}\text { Lantana camara } \\
\text { Linn. }\end{array}$ & Sadancing & $\mathrm{L}$ & - & - & $2+$ & 50 \\
\hline 48 & $\begin{array}{l}\text { Violaceae } \\
\text { Rinorea sp. } \\
\text { Vitaceae }\end{array}$ & Kayu pecat & $\mathrm{L}$ & - & $1+$ & $1+$ & $>1000$ \\
\hline 49 & Cissus sp. & Akar kudang & $\mathrm{L}$ & - & $1+$ & $2+$ & $>1000$ \\
\hline 50 & Vitis sp. & Daun katak & $\mathrm{L}$ & - & $1+$ & $2+$ & $>1000$ \\
\hline & Zingiberaceae & & & & & & \\
\hline 51 & Achasma foetens Val. & Puar angit & $\mathrm{L}$ & - & $2+$ & $3+$ & $>1000$ \\
\hline 52 & $\begin{array}{l}\text { Costus rumphianus } \\
\text { Linn. }\end{array}$ & Setawal & $\mathrm{L}$ & - & $1+$ & $2+$ & $>1000$ \\
\hline 53 & Costus sericeus $\mathrm{Bl}$. & Lempung puar & $\mathrm{L}$ & - & $1+$ & $1+$ & $>1000$ \\
\hline 54 & Costus sp. & Daun layang & $\mathrm{L}$ & - & $1+$ & $1+$ & $>1000$ \\
\hline 55 & Costus speciosus Sm. & Setawau & $\mathrm{L}$ & $1+$ & $1+$ & $3+$ & $>1000$ \\
\hline 56 & Globa maculata Bl. & Puar emas & $\mathrm{L}$ & - & $1+$ & $2+$ & $>1000$ \\
\hline 57 & $\begin{array}{l}\text { Riedelia carviflora } \\
\text { Oliv. }\end{array}$ & Puar angit & $\mathrm{L}$ & - & $1+$ & $2+$ & $>1000$ \\
\hline
\end{tabular}

${ }^{*}$ L: Leaves; $R$ : Root 
Medicinal herbs of Pasir Mayang, Jambi - Hilman Affandi et al.

\section{Alkaloids}

Of the 57 plant species, three species gave strong positive reaction (3+ - 4+) to alkaloid. Those species were Fibraurea sp , Zizyphus sp., and Uncaria sp. (Table 5).

Table 5. Plants giving strong positive reaction to Alkaloids

\begin{tabular}{ll}
\hline \multicolumn{1}{c}{ Family } & Species \\
\hline Menispermaceae & - Fibraurea sp. \\
Rhamnaceae & - Zizyphus sp. \\
Rubiaceae & - Uncaria sp. \\
\hline
\end{tabular}

\section{Saponins}

Of the 57 species tested for positive reaction to saponins, 12 species gave a strong positive reaction (3+ - 4+) (Table 6). Saponin is particularly prevalent in Bridelia glauca, B. minutiflora, Macaranga triloba, Callophylum mucumense, Litsea aurea, Barringtonia racemosa, magnolia mackottii, Pternandra coerulescens, Ficus variegata, Eugenia sp., Arenga pinnata, calamus sp., Miletia sp., Mucuna gigantea, Xanthophyllum sp., Uncaria sp., Euphoria malaiensis, Payena acuminata, Lygodium circinatum, Trema orientalis, and Lantana camara.

Table 6. Plants giving strong positive reaction to Saponins

\begin{tabular}{ll}
\hline \multicolumn{1}{c}{ Family } & \multicolumn{1}{c}{ Species } \\
\hline Amaranthaceae & Calosia argentea Linn \\
Asteraceae & Blumea balsamifera DC. \\
Cyatheaceae & Hemitelia latebrosa \\
Dioscoreaceae & Dioscorea aculeata Linn. \\
Piperaceae & Piper betle Linn. \\
Polypodiaceae & Dryopteris boryana O.Chr. \\
Rhyzoporaceae & Anisophyllea disticha Baill. \\
Rubiaceae & Lxora javanica DC. \\
Schizophyceaceae & Selaginella plana Hieron \\
Solanaceae & Solanum torvum Sw. \\
Verbenaceae & Clerodendron inerma Gaertn. \\
Zingiberaceae & Achasma foetens Val. \\
\hline
\end{tabular}




\section{Steroids/triterpenes}

Almost all plant species collected indicated positive response to steroids/ terpenes. Nine plant species showed strong reaction with $3+$ or $4+$ rating (Table 7). le compounds are particularly prevalent in Mallotus paninlatus; Clochidion •borescens; Litsea monopetala; Dictyopteris irregularis; Selaginella hieron.

Table 7. Plants giving strong positive reaction to steroids/triterpenes

\begin{tabular}{ll}
\hline \multicolumn{1}{c}{ Family } & \multicolumn{1}{c}{ Species } \\
\hline Asclepidirceae & Cryptolepis javanica Bl. Hoya sp. \\
Asteraceae & Blumea balsamifera DC. \\
Piperaceae & Piper aduncum Lind. \\
Polypodiaceae & Aspodium ternatense A.v.R. \\
Solanaceae & Solanum torvum Sw. \\
Zingiberaceae & Achasma foetens Val. \\
& Costus speciosus Sm. \\
\hline
\end{tabular}

\section{Toxicity tests}

Brine shrimp (Artemia salind) lethality test is a simple, fast and inexpensive oassay in the search for bioactive compounds from plant extracts. The value of ore than $1000 \mathrm{ppm}$ is considered non-lethal and hence shown as negative (-ve). A tal of more than 57 samples were tested and only ten showed toxicity against brine rimps. Of the ten plant species tested, three species (Dictiopteris irregularis Pr., intana camara Linn, and Gleichenia imearis Clarke) indicated some potential xicity which will require further investigation (Table 8).

Table 8. Lethal Concentration of Methanolic Extracts in the Brine Shrimp Larvae

\begin{tabular}{llc}
\hline \multicolumn{1}{c}{ Family } & \multicolumn{1}{c}{ Species } & $\begin{array}{c}\text { Toxicity } \\
\text { LC } 5 \text { (i.c.95\%) }\end{array}$ \\
Amaranthaceae & Calosia argentea Linn. & 645 \\
Asclepidirceae & Hoya sp. & 875 \\
Gleicheniaceae & Gleichenia linearis Clarke & 143 \\
Menispermaceae & Fibraurea sp. & 608 \\
Piperaceae & Piper sp. & 295 \\
Polypodiaceae & Dictyopteris irregularis Pr. & 2 \\
Rubiaceae & Uncaria sp. & 654 \\
Schizophyceaceae & Lygodium circinatum Sw. & 999 \\
Verberaceae & Clerodendron inerma Gaertn. & 282 \\
& Lantana camara Linn. & 50 \\
\hline
\end{tabular}


Medicinal herbs of Pasir Mayang, Jambi - Hilmau Affandi et al.

\section{REFERENCES}

Affandi, H., Nuryadin, A., Susilo.B.P and Supriatno. 1996. Phytochemical Survey of the Forest Tree Species of Pasir Mayang, Jambi, Sumatera, SEAMED BIOTROP, Research report.

Culvenor, C.C.J. arid J.S. Fitzgerald. 1963. A Field Method for Alkaloid Screening of Plants. J. Pharm. Sci. 52:303304.

Hostettmann, K., ed. 1991. Method in Plant Biochemistry, Vol 6 : Assays for Bioactivity, Pergamon Press, New York.

MeyerB.N., N.R. Ferrigni, J.E. Putman, L.B.Jacobsen, D.E. Nichol and J.L. McLaughli. 1982 Brine Shrimp: A Convenient General Bioassay for Active Plant Constituents. Planta Medica, 45:31-34

Moore, P.O. 1994. "Trials in bad taste." Nature 370:410-411.

Simes, J.J.H., J.G. Tracey, LJ. Webb and DJ. Dunstan. 1959. Australian Phytochemical Survey IH, Saponins in Eastern Australian Flowering Plants. Bulletin No. 281 C.S.I.R.O., Australia, Melbourne. 
Appendix 1. Traditional medicinal plants utilized by the population of Pasir Mayang village, subdistrict of VII Koto, District of Tebo, Jambi Province, Sumatera, Indonesia

\begin{tabular}{ccc}
\hline \hline $\begin{array}{c}\text { Species } \\
\text { (Voucher specimen) }\end{array}$ & Local name Part used & Uses \\
\hline
\end{tabular}

\section{Acanthaceae}

Justicia gendarusa L. setajam HA-259

\section{Amaranthaceae}

Calosia argentea Linn. HA-220

\section{Amaryllidaceae Curculigo capitulata O.K. HA-41}

Curculigo latifolia Dryand. benit HA-228

\section{Asclepidirceae}

Cryptolepis javanica $\mathrm{Bl}$.

HA-55

\section{Hoya sp.}

HA-136

\section{Asteraceae \\ Blumea balsamifera DC \\ HA-233}

\section{Burseraceae}

Canarium littorale $\mathrm{B} 1$.

HA-157 bunga merah

\author{
ketari; katorih Leave \\ bareh
}

akar daun taun Leaves

daun setebal Leaves

celapo tai

Leaves

The leaves are crushed, mixed with water, and filtered. The filtrate is administered to a recovering mother to strengthen and recondition her body.

ampelas licin

Roots
The root is squeezed and the liquid is adminis- tered as a headache and fever medication. Also used to relieve stomach aches.

\section{Connaraceae \\ Roureopsis javanica Planc \\ akar jaring}

HA-222 
Appendix 1. Continued

\begin{tabular}{|c|c|c|c|}
\hline $\begin{array}{c}\text { Species } \\
\text { (Voucher specimen) }\end{array}$ & Local name & Part used & Uses \\
\hline $\begin{array}{l}\text { Cyatheaceae } \\
\text { Hemitelia latebrosa Mett. } \\
\text { HA-58 }\end{array}$ & pakis tonggak & Leaves & $\begin{array}{l}\text { The leaves are crushed and mixed with } \\
\text { turmeric and rice. The mixture is rubbed on } \\
\text { the affected parts of the body to treat swelling. } \\
\text { The mixture is also rubbed on the stomach as a } \\
\text { stomach ache medication. }\end{array}$ \\
\hline $\begin{array}{l}\text { Dioscoreaceae } \\
\text { Dioscorea aculeata } \mathrm{L} . \\
\text { HA-221 }\end{array}$ & kabenar & Bulb & $\begin{array}{l}\text { Below-ground bulbs are eaten as a remedy for } \\
\text { mumps. }\end{array}$ \\
\hline $\begin{array}{l}\text { Euphorbiaceae } \\
\text { Melanolepis multiglandulosa } \\
\text { Reich.ex Zoll. } \\
\text { HA-270 }\end{array}$ & $\begin{array}{l}\text { bunga } \\
\text { sepanggil }\end{array}$ & Leaves & $\begin{array}{l}\text { The leaves are crushed, boiled, and cooled } \\
\text { down. The filtrate is used for babies to bathe } \\
\text { in for health promotion. }\end{array}$ \\
\hline $\begin{array}{l}\text { Gleicheniaceae } \\
\text { Gleichenia linearis Clarke } \\
\text { HA- } 84\end{array}$ & resam & Leaves & $\begin{array}{l}\text { The leaves are boiled and the decoction is } \\
\text { administered as a remedy for fever and ulcers. }\end{array}$ \\
\hline $\begin{array}{l}\text { Graminaceae } \\
\text { Centotheca latifolia Trin. } \\
\text { HA-255 }\end{array}$ & $\begin{array}{l}\text { rambut pulut- } \\
\text { pulut }\end{array}$ & Fruit & $\begin{array}{l}\text { The fruits are crushed and rubbed on the } \\
\text { swollen parts as a remedy against abscess. }\end{array}$ \\
\hline Driockloa djainkareh Busi & Buluh hitam & Leaves & $\begin{array}{l}\text { The leaves are crushed and filtered. The } \\
\text { filtrate is administered as appetite stimulant. }\end{array}$ \\
\hline $\begin{array}{l}\text { Labiatae } \\
\text { Ocimum basilicum } \mathrm{L} \text {. }\end{array}$ & selasih A & Leaves & $\begin{array}{l}\text { The leaves are sun-dried. The dried leaves are } \\
\text { crushed, mixed with coconut oil, and rubbed } \\
\text { on the affected parts to reduce swelling. }\end{array}$ \\
\hline $\begin{array}{l}\text { Leguminosae } \\
\text { Cassia alata } \mathrm{L} \text {. }\end{array}$ & ketepeng & Leaves & $\begin{array}{l}\text { Leaves are sun-dried. The dried leaves are } \\
\text { crushed, mixed with water, and rubbed on the } \\
\text { affected parts to cure skin diseases such as } \\
\text { scabies. }\end{array}$ \\
\hline Milletia sricea W.et & $\begin{array}{l}\text { Akar } \\
\text { semambu }\end{array}$ & Leaves & $\begin{array}{l}\text { The leaves are crushed and filtered. The } \\
\text { filtrate is administered as obat cacing perut or } \\
\text { purgative. }\end{array}$ \\
\hline
\end{tabular}


Appendix 1. Continued

\begin{tabular}{|c|c|c|c|}
\hline $\begin{array}{c}\text { Species } \\
\text { (Voucher specimen) }\end{array}$ & Local name & Part used & Uses \\
\hline Mucuna gigantean DC. & Akar katai & Root & $\begin{array}{l}\text { The roots are crushed and rubbed on the } \\
\text { stomach to cure stomach upset. (perut } \\
\text { kembung). }\end{array}$ \\
\hline $\begin{array}{l}\text { Liliaceae } \\
\text { Dianella montana } \mathrm{BL} \\
\text { HA-35 }\end{array}$ & $\begin{array}{l}\text { batang sia; } \\
\text { akar sayak }\end{array}$ & $\begin{array}{l}\text { Root; } \\
\text { Fruit }\end{array}$ & $\begin{array}{l}\text { The roots are sun-dried, boiled with water, and } \\
\text { used for bathing a recovering mother to } \\
\text { strengthen and recondition her body. The fruit } \\
\text { is eaten to reduce a swollen part of the body or } \\
\text { any other abscesses. }\end{array}$ \\
\hline $\begin{array}{l}\text { Smilax leucophylla B1. } \\
\text { HA-190 }\end{array}$ & akar gunung & Leaves & $\begin{array}{l}\text { The leaves are boiled and the decoction is } \\
\text { administered to stimulate appetite. }\end{array}$ \\
\hline $\begin{array}{l}\text { Menispermaceae } \\
\text { Fibraurea sp. } \\
\text { HA-93 }\end{array}$ & akar kunyit & Root & $\begin{array}{l}\text { The chopped roots are mixed with water and } \\
\text { applied externally to the eyes to cure } \\
\text { conjunctivitis. }\end{array}$ \\
\hline $\begin{array}{l}\text { Pericamphylus glaucus Merr. } \\
\text { HA-97 }\end{array}$ & $\begin{array}{l}\text { akar } \\
\text { limpanang; } \\
\text { akar manang; } \\
\text { akar benang }\end{array}$ & $\begin{array}{l}\text { Leaves; } \\
\text { Root }\end{array}$ & $\begin{array}{l}\text { The leaves of Pericamphylus glaucus and the } \\
\text { bark of Pometia pinnatas are boiled and the } \\
\text { decoction is administered as a fever medica- } \\
\text { tion. The root of } P \text {. glaucus, the bark of } P \text {. } \\
\text { pinnata, and rice are crushed and filtered. The } \\
\text { mixture is rubbed on the body also as a fever } \\
\text { medication. }\end{array}$ \\
\hline $\begin{array}{l}\text { Monimiaceae } \\
\text { Kibara } \mathrm{sp} \text {. } \\
\text { HA- } 103\end{array}$ & udu kuro & Leaves & $\begin{array}{l}\text { The leaves are heated on the fire to wither and } \\
\text { rubbed on the stomach to treat intestinal } \\
\text { worms. }\end{array}$ \\
\hline $\begin{array}{l}\text { Piperaceae } \\
\text { Piper aduncum L. } \\
\text { HA-15 }\end{array}$ & kayu sirih & Leaves & $\begin{array}{l}\text { The leaves are crushed and filtered. The } \\
\text { filtrate is used as eye drops. }\end{array}$ \\
\hline $\begin{array}{l}\text { Piper betle L. } \\
\text { HA- } 248\end{array}$ & sirih & Leaves & $\begin{array}{l}\text { The leaves are boiled and the decoction is } \\
\text { administered as a cough medicine. }\end{array}$ \\
\hline $\begin{array}{l}\text { Piper sp. } \\
\text { HA-30 }\end{array}$ & $\begin{array}{l}\text { akar sirih } \\
\text { duduk }\end{array}$ & Leaves & $\begin{array}{l}\text { The leaves are boiled and used to bathe a new } \\
\text { born baby to prevent fever. }\end{array}$ \\
\hline $\begin{array}{l}\text { Piper sp. } \\
\text { HA-242 }\end{array}$ & sirih antu & Leaves & $\begin{array}{l}\text { The leaves are roasted and rubbed on the } \\
\text { affected parts to relieve pain. }\end{array}$ \\
\hline
\end{tabular}


Appendix 1. Continued

\begin{tabular}{|c|c|c|c|}
\hline $\begin{array}{c}\text { Species } \\
\text { (Voucher specimen) }\end{array}$ & Local name & Part used & Uses \\
\hline $\begin{array}{l}\text { Polypodiaceae } \\
\text { Aspodium ternatense A.vR. }\end{array}$ & kelakap & Leaves & $\begin{array}{l}\text { The leaves are crushed and filtered. The filter } \\
\text { is drunk as a cure for fever }\end{array}$ \\
\hline $\begin{array}{l}\text { Dictyopteris irregularis } \operatorname{Pr} . \\
\text { HA-12 }\end{array}$ & $\begin{array}{l}\text { paku lipan/ } \\
\text { paku limat }\end{array}$ & Leaves & $\begin{array}{l}\text { The leaves are crushed and rubbed on the } \\
\text { stomach as a remedy for stomach aches }\end{array}$ \\
\hline $\begin{array}{l}\text { Dryopteris boryana O.Chr. } \\
\text { HA-31 }\end{array}$ & $\begin{array}{l}\text { pakis tonggak; } \\
\text { pakis longgoh }\end{array}$ & Leaves & $\begin{array}{l}\text { The leaves, mixed with turmeric and hulled } \\
\text { rice are crushed and mixed with water. The } \\
\text { concoction is rubbed on the stcmach as } \\
\text { stomach ache medication }\end{array}$ \\
\hline $\begin{array}{l}\text { Lomariopsis spectabilis } \\
\text { HA-56 }\end{array}$ & pakis gajah & Root & $\begin{array}{l}\text { Buttress roots are chopped, dried, and mixed } \\
\text { with the crushed bark of Millettia } \\
\text { atropurpurea and Pometia pinnata. The } \\
\text { mixture is soaked in warm water and rubbed } \\
\text { on the entire body to cure chicken pox }\end{array}$ \\
\hline $\begin{array}{l}\text { Nephrolepis hirsutula Presl. } \\
\text { HA-135 }\end{array}$ & pakis & Leaves & $\begin{array}{l}\text { The crushed leaves are used to treat abscesses } \\
\text { by rubbing on affected parts. Young leaves are } \\
\text { used as a vegetable }\end{array}$ \\
\hline $\begin{array}{l}\text { Rhamnaceae } \\
\text { Zizyphus sp. } \\
\text { HA-249 }\end{array}$ & akar kait & Stem & $\begin{array}{l}\text { The stem is chopped and the exuded liquid is } \\
\text { used as a gargle against oral ulceration. }\end{array}$ \\
\hline $\begin{array}{l}\text { Rhyzoporaceae } \\
\text { Anisophyllea disticha Baill. } \\
\text { HA-33 }\end{array}$ & batang beribu & Leaves & $\begin{array}{l}\text { The leaves are boiled and the decoction is } \\
\text { drunk as a fever medication }\end{array}$ \\
\hline $\begin{array}{l}\text { Rubiaceae } \\
\text { Lxora javanica DC. }\end{array}$ & $\begin{array}{l}\text { Kayu telur } \\
\text { ikan }\end{array}$ & Leaves & $\begin{array}{l}\text { The leaves are crushed and filtered. The } \\
\text { filtrate is drunk as a fever medication }\end{array}$ \\
\hline $\begin{array}{l}\text { Uncaria sp. } \\
\mathrm{HA}-45\end{array}$ & akar kait & Leaves & $\begin{array}{l}\text { The leaves are boiled and the liquid is } \\
\text { administered as a fever medication }\end{array}$ \\
\hline $\begin{array}{l}\text { Uncaria sp. } \\
\text { HA- }\end{array}$ & $\begin{array}{l}\text { akar kuku } \\
\text { alang }\end{array}$ & Leaves & $\begin{array}{l}\text { The leaves are crushed and rubbed on the } \\
\text { stomach to cure stomach ache }\end{array}$ \\
\hline
\end{tabular}


Appendix 1. Continued

\begin{tabular}{|c|c|c|c|}
\hline $\begin{array}{c}\text { Species } \\
\text { (Voucher specimen) }\end{array}$ & Local name & Part used & Uses \\
\hline
\end{tabular}

Rutaceae
Clausena excavata Burm
HA-34
Schizophyceaceae
Lygodium circinatum Sw.
HA-20
Selaginellaceae
Selaginella plana Hieron
HA-139

Rutaceae

Clausena excavata Burm

daun secere;

angit; puar

anjali

akar kawe ; akar Leaves

sapoi ; akar

menang

sigagar tegak; Leaves paku anai-anai ; nganai duduk

dubang

Solanaceae

Solanum torvum Sw.

HA-276

\section{Verbenaceae \\ Clerodendron inerma \\ Gaertn. HA-274}

Clerodendron squamatum Vahl.

HA-146

bunga pekan

Flower cere-cere; kayu

Leaves The leaves are crushed, mixed with water and table salt, and filtered. The filtrate is administered as a cure for stomach ache.

The leaves are crushed, mixed with water, and filtered. The residue is dried, cooked with water, and eaten as a fever medication.

The leaves are boiled and the decoction is used to bathe three times a day to cure jaundice.

The leaves are crushed and rubbed on the stomach as a remedy for diarrhea.

Fruit The fruit is eaten as a vegetable.

The fruits are boiled and the decoction is administered as a fever medication.

The flowers, mixed with the flower of Calosia argentea, are crushed and rubbed on a baby as a fever medication.

Leaves The leaves mixed with turmeric are crushed and filtered. The filtrate is used to bathe the babies in case of fever.

Stem The twigs are chopped and boiled. The decoction is administered to cure stomach aches.

Leaves The leaves are boiled and the decoction is administered as a poison antidote.

Rinorea sp.

HA-179

Vitaceae

akar kudang

Leaves

Cissus sp.

HA-134

Vitis sp.

HA-107 kayu pecat

sadancing

daun katak
Leaves
The leaves are boiled and the decoction is drunk to relieve headaches.

The leaves are crushed, mixed with water, and rubbed on the entire body to cause flatulence. 
Appendix 1. Continued

\begin{tabular}{|c|c|c|c|}
\hline $\begin{array}{c}\text { Species } \\
\text { (Voucher specimen) }\end{array}$ & Local name & Part used & Uses \\
\hline $\begin{array}{l}\text { Zingiberaceae } \\
\text { Achasma foetens Val. } \\
\text { HA-13 }\end{array}$ & puar angit & Leaves & $\begin{array}{l}\text { The leaves are chopped, soaked in water and } \\
\text { the decoction is administered as a fever } \\
\text { medication }\end{array}$ \\
\hline $\begin{array}{l}\text { Costus rumphianus } \mathrm{L} \text {. } \\
\text { HA-216 }\end{array}$ & setawar & $\begin{array}{l}\text { Leaves; } \\
\text { Stem }\end{array}$ & $\begin{array}{l}\text { The leaves are chopped, soaked in water, and } \\
\text { rubbed on the waist and ribs to treat aches and } \\
\text { pains. }\end{array}$ \\
\hline & & & $\begin{array}{l}\text { The stem is chopped, boiled with table salt, } \\
\text { and drunk to treat whooping cough }\end{array}$ \\
\hline $\begin{array}{l}\text { Costus sericeus } \mathrm{B} 1 \text {. } \\
\mathrm{HA}-11\end{array}$ & limpung puar & Leaves & $\begin{array}{l}\text { The leaves are crushed and applied on the } \\
\text { fore-head to treat headaches }\end{array}$ \\
\hline $\begin{array}{l}\text { Costus sp. } \\
\text { HA-38 }\end{array}$ & daun layang & Leaves & $\begin{array}{l}\text { The leaves are boiled, and the decoction is } \\
\text { administered as a fever medication. }\end{array}$ \\
\hline $\begin{array}{l}\text { Costus speciosus } \mathrm{Sm} \\
\mathrm{HA}-23\end{array}$ & setawau & Stem & $\begin{array}{l}\text { The stem is squeezed and the extract is } \\
\text { adminis-tered as a cough medicine }\end{array}$ \\
\hline $\begin{array}{l}\text { Globa maculata } \text { Bl. } \\
\text { HA-66 }\end{array}$ & puar emas & Leaves & $\begin{array}{l}\text { The leaves, mixed with the leaves of } \\
\text { Melastoma malabathricum, are boiled and the } \\
\text { decoction is administered as a fever } \\
\text { medication }\end{array}$ \\
\hline
\end{tabular}

